\title{
AN EXTENED PHANTOM NODE METHOD FOR CRACK INTERACTIONS IN COMPOSITES
}

\author{
B. Chen ${ }^{1,2}$, S. T. Pinho ${ }^{1}$, P. M. Baiz ${ }^{1}$, T. E. Tay ${ }^{2}$ \\ ${ }^{1}$ Department of Aeronautics, Imperial College London (b.chen09@imperial.ac.uk) \\ ${ }^{2}$ Department of Mechanical Engineering, National University of Singapore
}

\begin{abstract}
Failure of fibre-reinforced composite materials is usually preceded by the development of a network of cracks running through plies (matrix cracks) and between plies (delamination). Matrix cracks and delamination often interact with each other to form complex crack paths. A novel numerical method is developed to model all plies of the laminate by one element. Matrix cracks, delamination and matrix crack tips at the interface can all be explicitly represented within such an element to achieve high accuracy modelling of the mechanism of interaction. A $2 D$ element formulated based on this method has demonstrated good performance on the delamination/matrix crack interaction problem in a cross-ply composite laminate.
\end{abstract}

Keywords: phantom node method, multiple cracks, matrix crack, delamination.

\section{INTRODUCTION}

The failure of composite materials is known for its complexity in nature. Unlike other materials in engineering practice such as metal or concrete, the ultimate failure of composites is usually preceded by a high count of matrix cracks in plies and delamination between plies. Recent experiments in the literature [1] have shown that during in-plane loading situations, matrix cracks can induce widespread delamination which destroys the structural integrity of the laminate much earlier than the fibre-breaking of the 0-plies. In such cases, the matrix crack/delamination interaction is critical for the failure of the laminate. The work by van der Meer et al. [2] has used a phantom node method to explicitly represent matrix cracks which is shown to be important for accurate predictions on the matrix crack/delamination interaction. Fang et al. [3] have shown that the representation of matrix crack tips at the interface is also important for the prediction of the stress concentration at the intersection of matrix cracks and the interface (Figure 1). They then explicitly included the matrix crack tips in the interface by forming an augmented cohesive element based on the augmented finite element method proposed in [4] such that it can break into two parts with respect to the locations of matrix crack tips at the upper and lower plies.

In this work, a novel numerical method is developed from an extension of the phantom 
node method to model multiple cracks within one element. Section 2.1 will give a review of the phantom node method with an emphasis on the modelling of cohesive cracks. Section 2.2 will investigate the relation between the standard FEM and the phantom node method. Section 2.3 will propose the theory of the extended phantom node method based on the conclusion from section 2.2. Section 3 will show that an element based on this new method is capable of modelling all the plies and interfaces of a laminate inside one element such that the information of their failure status is shared among them. Matrix cracks, interfaces and the matrix crack tips at the interfaces are all explicitly represented within the element to achieve a high accuracy in the modelling of matrix crack/delamination interaction.

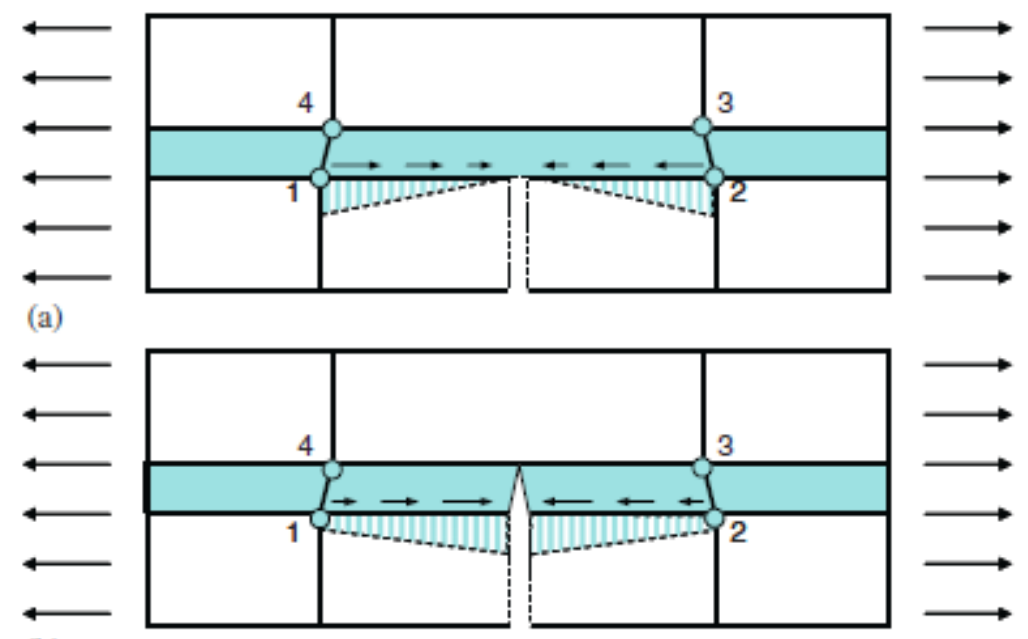

(b)

Figure 1. Stress field predictions at the interface: (a) without representing the matrix crack tip; (b) with the matrix crack tip explicitly represented [3].

\section{THEORY}

\subsection{Phantom node method}

The phantom node method was first proposed by Hansbo and Hansbo [5] and is later implemented and developed in [4, 6, 7]. In the phantom node method, a material domain with a potential internal discontinuity can be modelled by one element with two pair of nodes, namely real nodes and ghost nodes. When the stresses of the element reach the material strength, a discontinuity is modelled by forming two superposing elements with the help of ghost nodes (Figure 2). Each of the two elements contains only part of the material domain. Since all the nodes of the elements are at the outer material boundaries, the location of the discontinuity does not need to be known during meshing. When modelling a strong discontinuity (stress-free crack) inside an element, the phantom node method has been proven to be equivalent to the eXtended Finite Element Method (XFEM) with only the Heaviside enrichment function [6]. Both methods essentially use extra Degrees Of Freedom (DOFs) to interpolate the new crack surfaces. The difference between the two is that the phantom node method keeps the nodal DOFs as displacement DOFs and stores the extra DOFs needed as the dis- 
placement DOFs of the ghost nodes, while XFEM keeps the number of nodes constant and stores the extra DOFs needed as enriched DOFs at each node. The advantage of the former is that it is easier to be implemented in existing FEM programmes because each node has only the standard displacement DOFs and only the standard FEM shape functions are needed to interpolate them.

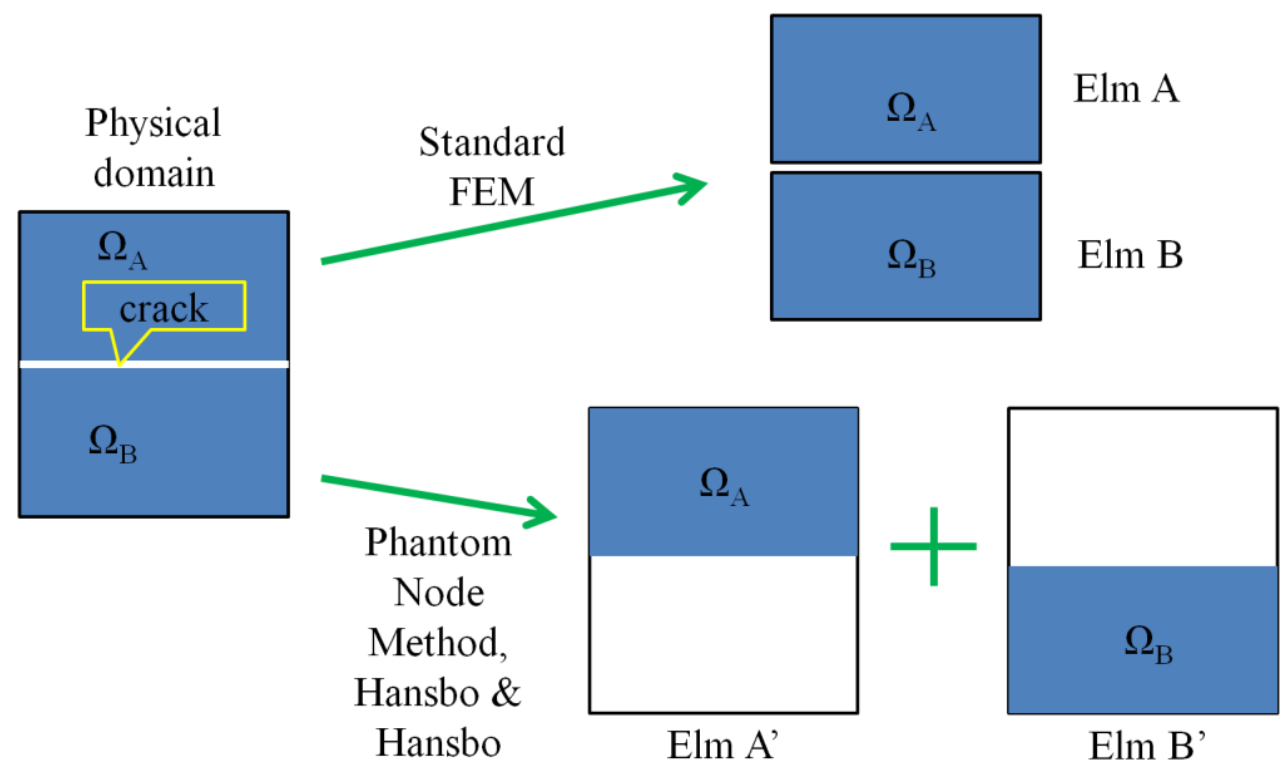

(2004)

Figure 2. Phantom node method

Currently the phantom node method has only been used to model one crack inside an element. Although some concept of modelling multiple cracks has been presented in [4], the detailed formulation and implementation remain absent in the literature. In particular, the current phantom node method has difficulties in modelling multiple cohesive cracks within one element. When modelling one cohesive crack, the phantom node method breaks the original element into two parts with the help of phantom nodes and modifies the equilibrium equation of the new material domain to include the energy contributions of the cohesive tractions on the new material boundaries $[4,6,7]$. In the absence of body forces, the equilibrium equation before the initiation of the cohesive crack is:

$$
\int_{\Omega} \delta \mathbf{\varepsilon}^{T} \boldsymbol{\sigma} \mathrm{d} V=\int_{S} \delta \mathbf{u}^{T} \mathbf{t} \mathrm{d} s
$$

where $\boldsymbol{\sigma}$ is the internal stress and $\mathbf{t}$ is the external force. $\delta \boldsymbol{\varepsilon}$ is the virtual strain and $\delta \mathbf{u}$ is the virtual displacement. When a cohesive crack develops at the surface $S_{C}$ and breaks the material domain into $\Omega_{A}$ and $\Omega_{B}$ respectively, the single equilibrium equation becomes a system of equations:

$$
\int_{\Omega_{A}} \delta \boldsymbol{\varepsilon}^{T} \boldsymbol{\sigma} \mathrm{d} V=\int_{S_{A}} \delta \mathbf{u}^{T} \mathbf{t} \mathrm{d} s+\int_{S_{C}^{A}} \delta \mathbf{v}^{T} \mathbf{t}^{c}(\mathbf{w}) \mathrm{d} s, \quad \int_{\Omega_{B}} \delta \boldsymbol{\varepsilon}^{T} \boldsymbol{\sigma} \mathrm{d} V=\int_{S_{B}} \delta \mathbf{u}^{T} \mathbf{t} \mathrm{d} s-\int_{S_{C}^{B}} \delta \mathbf{v}^{T} \mathbf{t}^{c}(\mathbf{w}) \mathrm{d} s
$$

where $\mathbf{t}^{\mathrm{c}}$ is the cohesive stress and $\mathbf{w}$ and $\delta \mathbf{v}$ are respectively the real and virtual displacement 
jumps across the crack surface. The two equations are coupled and they need to be rearranged and combined into one linear system before they can be used in a FEM program. When another cohesive crack is to be modelled, the equilibrium equation will need to be modified again to include the new cohesive force contributions. This would require considerable work to rearrange and combine the coupled equilibrium equations into one linear system. This work may be tedious especially when more cohesive cracks need to be modelled and they may not appear in a fixed sequence.

A novel method is needed to model multiple cohesive cracks in a more efficient way. The phantom node method is investigated and compared with the standard FEM in the next section and a new method is proposed to extend the current phantom node method to efficiently model multiple cohesive cracks within one element.

\subsection{Equivalence of FEM and phantom node method}

In this section, a detailed comparison between the solution procedures of the FEM and the phantom node method is investigated and it is found that the displacement solutions by the two methods are exactly the same under certain easily-satisfied conditions. Let's take the elements in Figure 2 as an example, we shall prove that the displacement solutions over $\Omega_{A}$ by the standard finite element Elm A and phantom node element Elm A' are exactly the same.

Firstly, we have the discretized equilibrium equation by standard FEM is

$$
[K]\{U\}=\{F\}
$$

where

$$
[K]=\int_{\Omega_{A}}[B]^{T}[D][B] d V, \quad\{F\}=\int_{S_{A}}[N]^{T}\{t\} d s
$$

and $[N]$ is the shape function matrix:

$$
[N]=\left[\begin{array}{ccccccc}
N_{1} & 0 & 0 & \cdots & N_{n} & 0 & 0 \\
0 & N_{1} & 0 & \cdots & 0 & N_{n} & 0 \\
0 & 0 & N_{1} & \cdots & 0 & 0 & N_{n}
\end{array}\right]
$$

and $N_{j} \mathrm{~s}$ are the standard FEM shape functions defined on $\Omega_{A} \cdot[B]$ is the deformation matrix:

$$
[B]=[L]^{T}[N], \quad[L]=\left[\begin{array}{cccccc}
\frac{\partial}{\partial x} & 0 & 0 & \frac{\partial}{\partial y} & \frac{\partial}{\partial z} & 0 \\
0 & \frac{\partial}{\partial y} & 0 & \frac{\partial}{\partial x} & 0 & \frac{\partial}{\partial z} \\
0 & 0 & \frac{\partial}{\partial z} & 0 & \frac{\partial}{\partial x} & \frac{\partial}{\partial y}
\end{array}\right]
$$

The nodal displacement vector $[U]$ is solved from (3) and the displacement solution on $\Omega_{A}$ by Elm A is obtained as: 


$$
\{u\}=[N]\{U\}
$$

The solution procedure for Elm A' is exactly the same as that of the Elm A. The only difference is that in Elm A' the displacement is interpolated with the shape functions having a support on $\Omega$ instead of on $\Omega_{A}$. Only the part of the shape functions restricted on $\Omega_{A}$ is used:

$$
\begin{gathered}
\left\{u^{\prime}\right\}=\left[\left.N^{\prime}\right|_{\Omega_{A}}\right]\left\{U^{\prime}\right\} \\
{\left[N^{\prime}\right]=\left[\begin{array}{ccccccc}
N_{1}^{\prime} & 0 & 0 & \cdots & N_{n^{\prime}}^{\prime} & 0 & 0 \\
0 & N_{1}^{\prime} & 0 & \cdots & 0 & N_{n^{\prime}}^{\prime} & 0 \\
0 & 0 & N_{1}^{\prime} & \cdots & 0 & 0 & N_{n^{\prime}}^{\prime}
\end{array}\right]}
\end{gathered}
$$

where $N_{i}^{\prime} \mathrm{s}$ are standard FEM shape functions defined on the domain $\Omega$. We can get the discretized equilibrium equation for Elm A':

$$
\begin{gathered}
{\left[K^{\prime}\right]\left\{U^{\prime}\right\}=\left\{F^{\prime}\right\}} \\
{\left[K^{\prime}\right]=\int_{\Omega_{A}}\left[B^{\prime}\right]^{T}[D]\left[B^{\prime}\right] d V, \quad\left\{F^{\prime}\right\}=\int_{S_{A}}\left[\left.N^{\prime}\right|_{\Omega_{A}}\right]^{T}\{t\} d s}
\end{gathered}
$$

with

$$
\left[B^{\prime}\right]=[L]^{T}\left[\left.N^{\prime}\right|_{\Omega_{A}}\right]
$$

$\left[K^{\prime}\right]$ is symmetric positive definite, therefore the nodal solution $\left\{U^{\prime}\right\}$ exists and it is unique. The critical question here is whether the displacement solutions over the material domain $\Omega_{A}$ by these two methods are the same, i.e. whether (7) and (8) are equivalent to each other. An important property of the standard FEM shape functions is that they form a basis for polynomials of the same order over their domain. Since $N_{j}$ form a basis of polynomials of the same order over $\Omega_{A}$, and $N_{i}^{\prime}$ are well defined over $\Omega_{A}$ and of the same order as $N_{j}$, we have the following relation:

$$
\left.N_{i}^{\prime}\right|_{\Omega_{A}}=\sum_{j=1}^{n} a_{j}^{i} N_{j}
$$

Similarly, $N_{i}^{\prime}$ form a basis of polynomials of the same order over $\Omega$ which contains $\Omega_{A}$, therefore $\left.N_{i}^{\prime}\right|_{\Omega_{A}}$ form a basis of polynomials of the same order over $\Omega_{A}$. A similar equation as (13) can be written:

$$
N_{i}=\left.\sum_{j=1}^{n} b_{j}^{i} N_{j}^{\prime}\right|_{\Omega_{A}}
$$

Therefore there is an invertible map $T$ between $\left.N_{i}^{\prime}\right|_{\Omega_{A}}$ and $N_{j}$ :

$$
\left[\left.N^{\prime}\right|_{\Omega_{A}}\right]=[N][T]
$$

where: 


$$
[T]=\left[\begin{array}{ccccccc}
a_{1}^{1} & 0 & 0 & \cdots & a_{1}^{n^{\prime}} & 0 & 0 \\
0 & a_{1}^{1} & 0 & \cdots & 0 & a_{1}^{n^{\prime}} & 0 \\
0 & 0 & a_{1}^{1} & \cdots & 0 & 0 & a_{1}^{n^{\prime}} \\
\vdots & \vdots & \vdots & \ddots & \vdots & \vdots & \vdots \\
a_{n}^{1} & 0 & 0 & \cdots & a_{n}^{n^{\prime}} & 0 & 0 \\
0 & a_{n}^{1} & 0 & \cdots & 0 & a_{n}^{n^{\prime}} & 0 \\
0 & 0 & a_{n}^{1} & \cdots & 0 & 0 & a_{n}^{n^{\prime}}
\end{array}\right]
$$

If we substitute the position vector of node $j$ on both sides of (13), we will get:

$$
\left.N_{i}^{\prime}\right|_{\Omega_{A}}\left(\mathbf{x}_{j}\right)=a_{j}^{i}
$$

Therefore, $a_{j}^{i}$ is the interpolation of the shape function of Elm $A$ ' node $I$ at the position of Elm $A$ node $j$.

Substitute (15) into (10) to (12) we can get

$$
\begin{gathered}
{\left[B^{\prime}\right]=[L]^{T}[N][T]} \\
{\left[K^{\prime}\right]=[T]^{T}[K][T], \quad\left\{F^{\prime}\right\}=[T]^{T}\{F\}} \\
{[T]^{T}[K][T]\left\{U^{\prime}\right\}=[T]^{T}\{F\}}
\end{gathered}
$$

Because $[T]$ is invertible, we have:

$$
[K][T]\left\{U^{\prime}\right\}=\{F\}
$$

Since $[K]$ is the standard FEM stiffness matrix which is symmetric positive definite, the solution of the system $[K]\{X\}=\{Y\}$ is unique. Therefore we have:

$$
[T]\left\{U^{\prime}\right\}=\{U\}
$$

Substituting (15) and (22) into (7), we can get:

$$
\{u\}=[N][T]\left\{U^{\prime}\right\}=\left[\left.N^{\prime}\right|_{\Omega_{A}}\right]\left\{U^{\prime}\right\}=\left\{u^{\prime}\right\}
$$

Therefore the displacement solutions over the material domain $\Omega_{A}$ by the two methods are exactly the same when the shape functions used in the two methods are polynomials of the same order and they are both defined over $\Omega_{A}$.

\subsection{Extended phantom node method}

Based on the conclusion in section 2.2, we can see that as long as the phantom element Elm A' contains the material domain $\Omega_{A}$, there is no other restriction on the positions of the nodes of Elm A' for it to have the same displacement solution as the standard finite element Elm A. The nodes of Elm A' in theory can be all outside of $\Omega_{A}$ and the equations in section 
2.2 would still hold and Elm A' would still behave exactly like Elm A (Figure 3). Therefore, a complete distinction between numerical domain and material domain can be made. An element can have all its nodes as ghost nodes, completely detached from the material domain that it interpolates on, and has exactly the same solution as a standard finite element on this material domain.

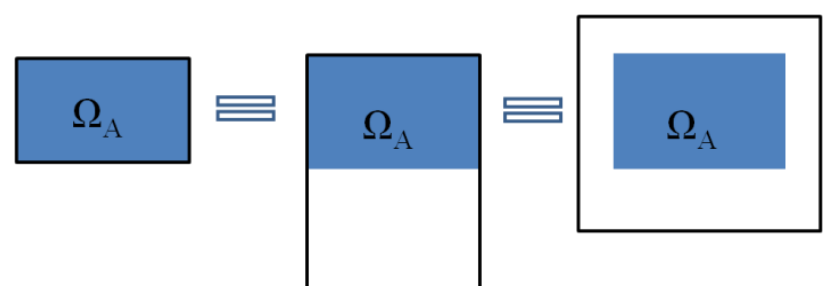

Figure 3. Complete distinction between the numerical domain and the material domain

From this observation, it can be claimed that for any standard FEM mesh, a phantom element with multiple pairs of nodes can be constructed such that it behaves exactly like this mesh. This can be done by finding the equivalent phantom element of each standard element in the FEM mesh and then group them together (Figure 4). The stiffness matrix of the phantom element can be obtained by following the same assembly procedure of the standard FEM mesh.

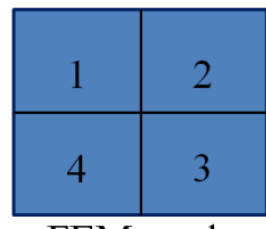

FEM mesh
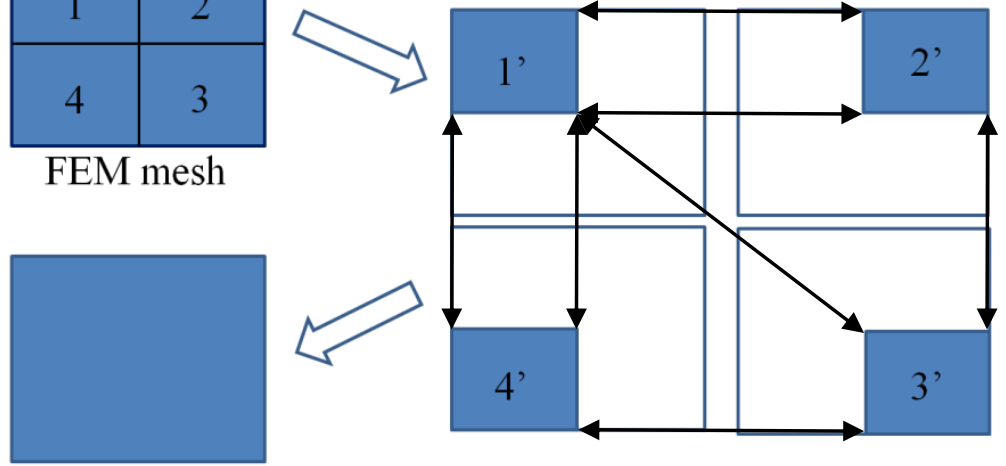

Phantom element

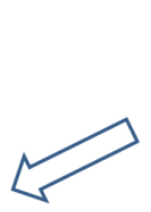

Figure 4. Construction of a phantom element based on a FEM mesh

This discovery greatly facilitates the modelling of cohesive cracks. When modelling one cohesive crack inside such a phantom element, the element is broken into three parts with the cohesive crack modelled independently by a phantom cohesive element (Figure 5). The cohesive force is represented as an internal force with a direct contribution to the internal energy of the phantom element. The equilibrium equations after the initiation of the cohesive crack are:

$$
\int_{\Omega_{A}} \delta \boldsymbol{\varepsilon}^{T} \boldsymbol{\sigma} \mathrm{d} V=\int_{S_{A}} \delta \mathbf{u}^{T} \mathbf{t} \mathrm{d} s, \quad \int_{\Omega_{B}} \delta \boldsymbol{\varepsilon}^{T} \boldsymbol{\sigma} \mathrm{d} V=\int_{S_{B}} \delta \mathbf{u}^{T} \mathbf{t} \mathrm{d} s, \quad \int_{S_{C}} \delta \mathbf{v}^{T} \mathbf{t}^{c}(\mathbf{w}) \mathrm{d} s=\int_{\partial S_{C}} \delta \mathbf{u}^{T} \mathbf{t} \mathrm{d} x
$$

The equilibrium equation of any of the three parts remains a standard finite element equilibrium equation. The stiffness matrix and the force vector of the phantom element after introducing the cohesive crack can be easily obtained by the standard finite element assembly 
procedure for a mesh of three elements. This enables the modelling of multiple cohesive cracks inside one element by simply adding more nodes at the initial element corners, without the need to modify the equilibrium equation as done in the phantom node method.

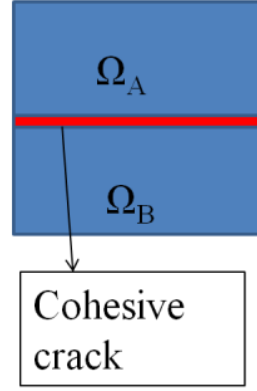

(a)
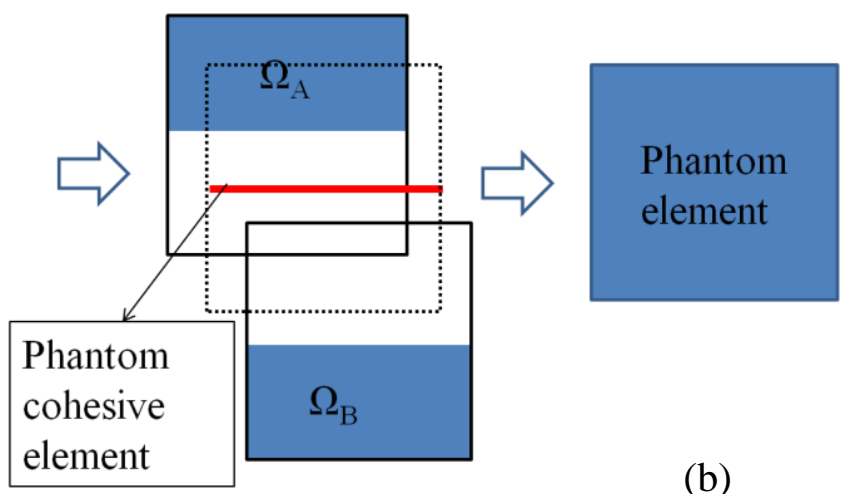

(b)

Figure 5. Modelling of a cohesive crack in a phantom element

\section{APPLICATIONS}

The method in section 2.3 is first employed in the modelling of a single cohesive crack inside one element. A standard FEM mesh as shown in Figure 5(a) with $\Omega_{A}$ and $\Omega_{B}$ modelled by two quadrilateral elements and the cohesive crack modelled by a cohesive element is built in Abaqus. A phantom element as shown in Figure 5(b) is built in an Abaqus User Element (UEL) subroutine. Based on the conclusion in section 2.2, the phantom element should behave exactly like the standard FEM mesh. A simple tension loading and a simple shear loading are performed on both the FEM mesh and the UEL phantom element. The stressdisplacement curves by the two methods are compared in Figure 6 and Figure 7. It can be seen that the two methods give identical response which supports the conclusion in section 2.2 .
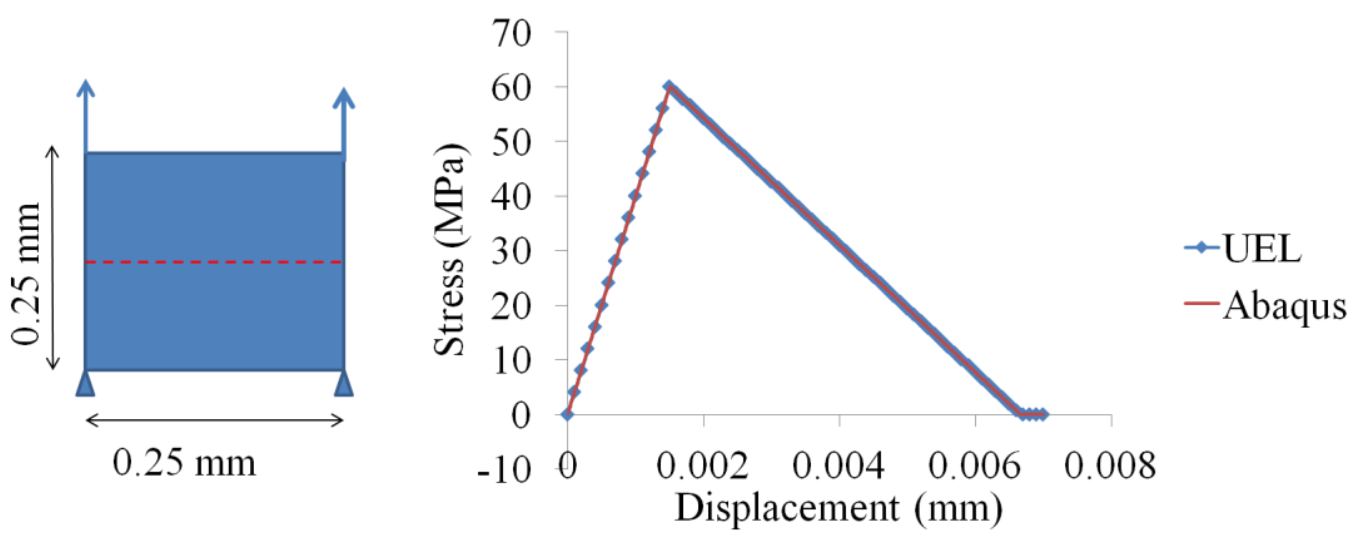

Figure 6. Simple tension loading 


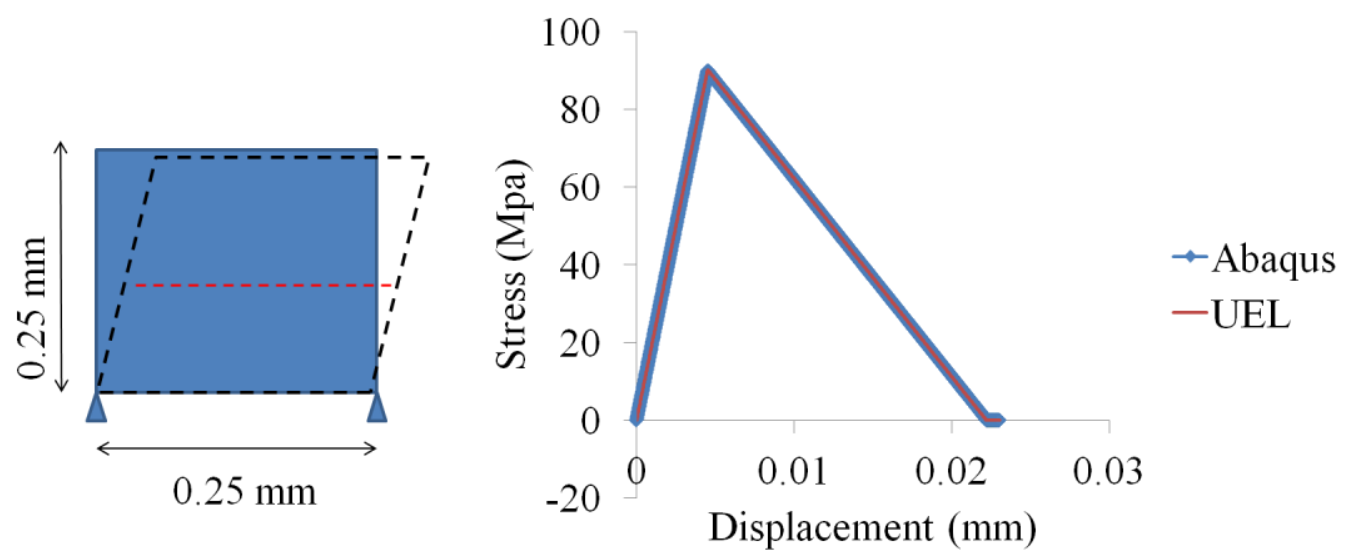

Figure 7. Simple shear loading

The method is then further employed on developing a phantom element with multiple pairs of ghost nodes to model multiple discontinuities within a cross-ply [90/0] composite laminate. The entire laminate is modelled within such an element. Initially, both plies of the laminate are intact and they are connected by an interface (Figure 8(a)). Since a cohesive element is used to model the interface, this element is capable of capturing the scenario where delamination occurs without any matrix crack.

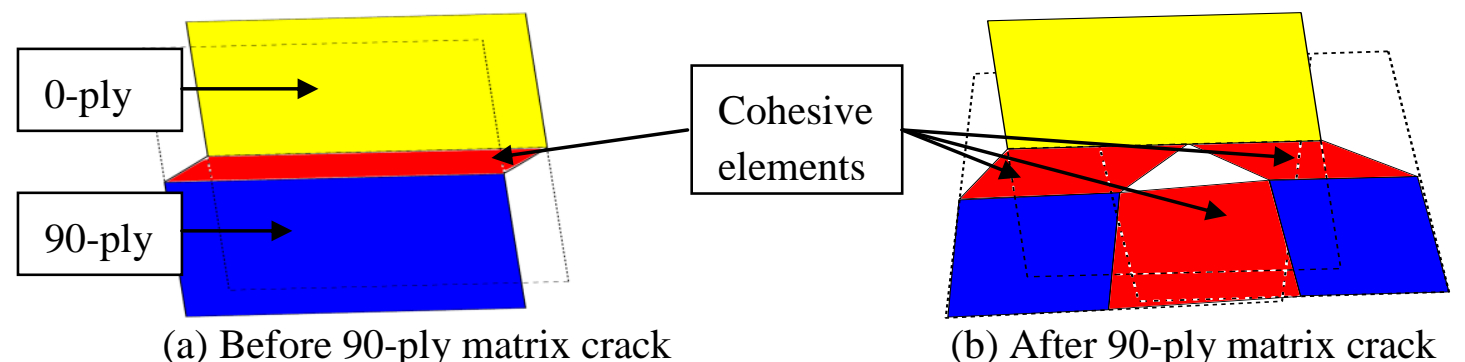

Figure 8. Cross-ply phantom element before and after matrix crack initiation (cohesive element thickness exaggerated).

The matrix crack/delamination interaction comes into play when the matrix crack occurs in the 90-ply. The stress concentration caused by the matrix crack tip at the interface often leads to delamination. In order to capture this stress concentration accurately, both the matrix crack and the crack tip are explicitly represented. When matrix crack occurs in the 90ply, the 90-ply element will break into two linear elements and one cohesive element with the help of extra ghost nodes. The original cohesive element at the interface will also break into two parts with respect to the position of the matrix crack tip (Figure 8(b)).

The phantom element is written in an Abaqus UEL subroutine. Post-processing is performed by a Matlab program. In order to verify the performance of the phantom element, a standard FEM mesh as shown in Figure 9(a) is built in Abaqus and the results of the two methods are compared. The FEM model here is very detailed for the scale of a laminate since the delamination, matrix crack and matrix crack tip are all explicitly represented. A simple tensile loading with symmetric boundary condition on the surface of the 0-ply is applied to both the Abaqus model and the phantom element (Figure 9). The material is assumed to be typical glass-epoxy composite. The predictions of matrix crack and delamination by the two 
methods are compared in Figure 10. For the phantom element, failure information is available only at the integration points of the cohesive elements. A red dot appears when there is failure. Both the deformation and the failure sequence predictions by the two methods are in good agreement with each other. Both methods predicted that initial failure occurs only in the 90ply with no delamination during the initial stage of loading. As the load increases, the stress concentration caused by the matrix crack tip at the interface eventually leads to the propagation of delamination at the interface and a ' $T$ ' shaped crack forms (Figure 10(b)). The predictions of the laminate stress-strain curve are compared in Figure 11. The two curves follow closely with each other. The slight change of slope due to the 90-ply failure can be observed.

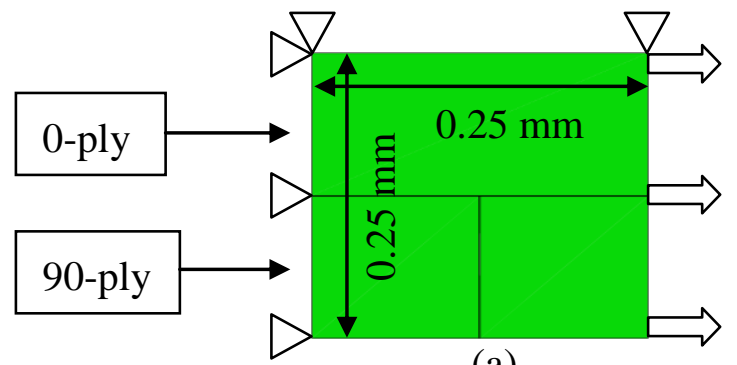

(a)

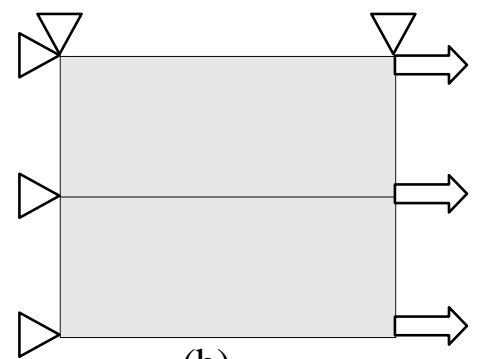

(b)

Figure 9. (a): Abaqus mesh: 3 quadrilateral elements and 3 cohesive elements (interface is represented by two cohesive elements); (b): UEL phantom element

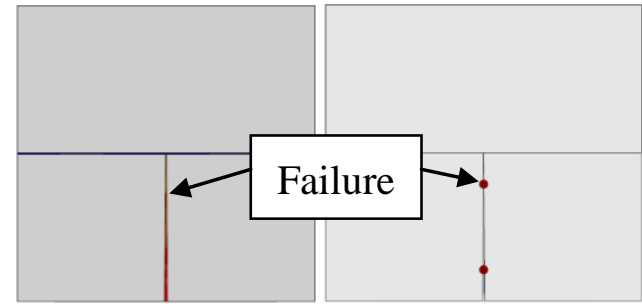

Abaqus
UEL

(a) Initial stage

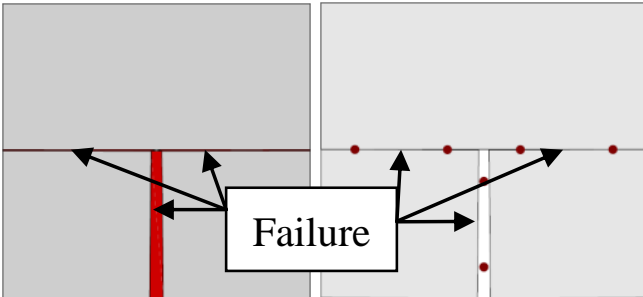

Abaqus
UEL

(b) Final stage

Figure 10. Comparison of failure predictions between Abaqus standard FEM model and UEL phantom element. Red colour indicates failure. Only the failure status at the integration points is displayed in the phantom element. 


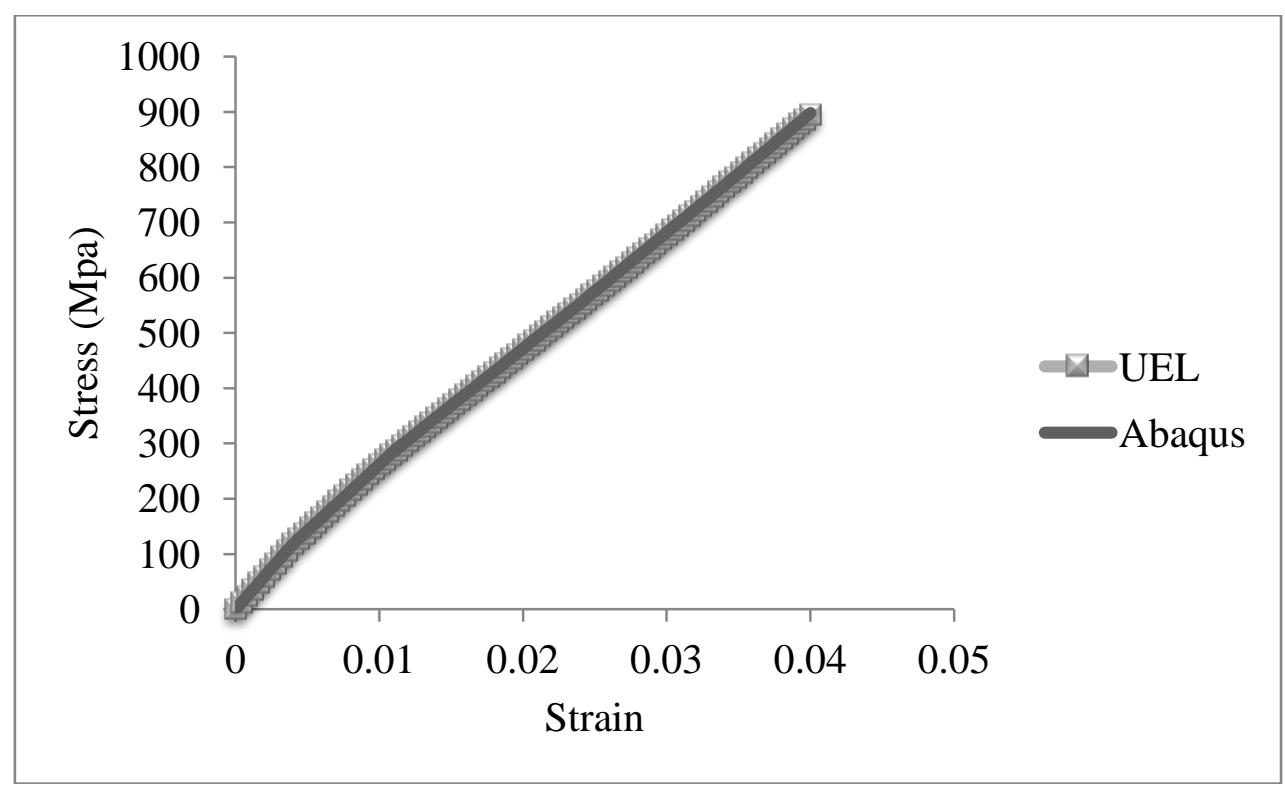

Figure 11. Stress-strain curves

It can be seen that the phantom element established based on the extended phantom node method is able to model the development of multiple cohesive cracks and it has the degree of accuracy as that of a detailed FEM mesh. In the extended phantom node method, the locations of all cracks do not need to be known before analysis. They can be modelled by activating extra numerical nodes during analysis when a failure criterion is met. The extended phantom node method can be easily extended to model more plies and more cracks with the help of extra ghost nodes.

\section{CONCLUSION}

A novel numerical method (the extended phantom node method) is proposed based on the theory of the phantom node method. The extended phantom node method greatly facilitates the modelling of multiple cohesive cracks inside one element. A phantom element based on this method is formulated to model the matrix crack/delamination interaction in a cross-ply laminate. The whole laminate is modelled in the element, with the delamination, matrix crack and matrix crack tip all explicitly represented. Good performance is observed on both the failure pattern and the stress-strain curve predictions by the phantom element.

\section{Acknowledgements}

The first author greatly acknowledges the research scholarship from National University of Singapore.

\section{REFERENCES}

[1] B. G. Green, M. R. Wisnom, and S. R. Hallett, "An experimental investigation into the 
tensile strength scaling of notched composites," Composites Part A-Applied Science and Manufacturing, vol. 38, no. 3, pp. 867-878, 2007.

[2] F. P. van der Meer, L. J. Sluys, S. R. Hallett et al., "Computational modeling of complex failure mechanisms in laminates," Journal of Composite Materials, vol. 46, pp. 603-623, 2011.

[3] X. J. Fang, Q. D. Yang, B. N. Cox et al., "An augmented cohesive zone element for arbitrary crack coalescence and bifurcation in heterogeneous materials," International Journal for Numerical Methods in Engineering, vol. 88, no. 9, pp. 841-861, 2011.

[4] D. S. Ling, Q. D. Yang, and B. Cox, "An augmented finite element method for modeling arbitrary discontinuities in composite materials," International Journal of Fracture, vol. 156, no. 1, pp. 53-73, 2009.

[5] A. Hansbo, and P. Hansbo, "A finite element method for the simulation of strong and weak discontinuities in solid mechanics," Computer Methods in Applied Mechanics and Engineering, vol. 193, no. 33-35, pp. 3523-3540, 2004.

[6] J.-H. Song, P. M. A. Areias, and T. Belytschko, "A method for dynamic crack and shear band propagation with phantom nodes," International Journal for Numerical Methods in Engineering, vol. 67, no. 6, pp. 868-893, 2006.

[7] F. P. van der Meer, and L. J. Sluys, "A phantom node formulation with mixed mode cohesive law for splitting in laminates," International Journal of Fracture, vol. 158, no. 2, pp. 107-124, 2009. 